\title{
Fast Continuous Collision Detection using Deforming Non-Penetration Filters
}

\author{
Min Tang* \\ Zhejiang University
}

\author{
Dinesh Manocha $^{\dagger}$ \\ University of North Carolina at Chapel Hill
}

\author{
Ruofeng Tong ${ }^{\ddagger}$ \\ Zhejiang University
}

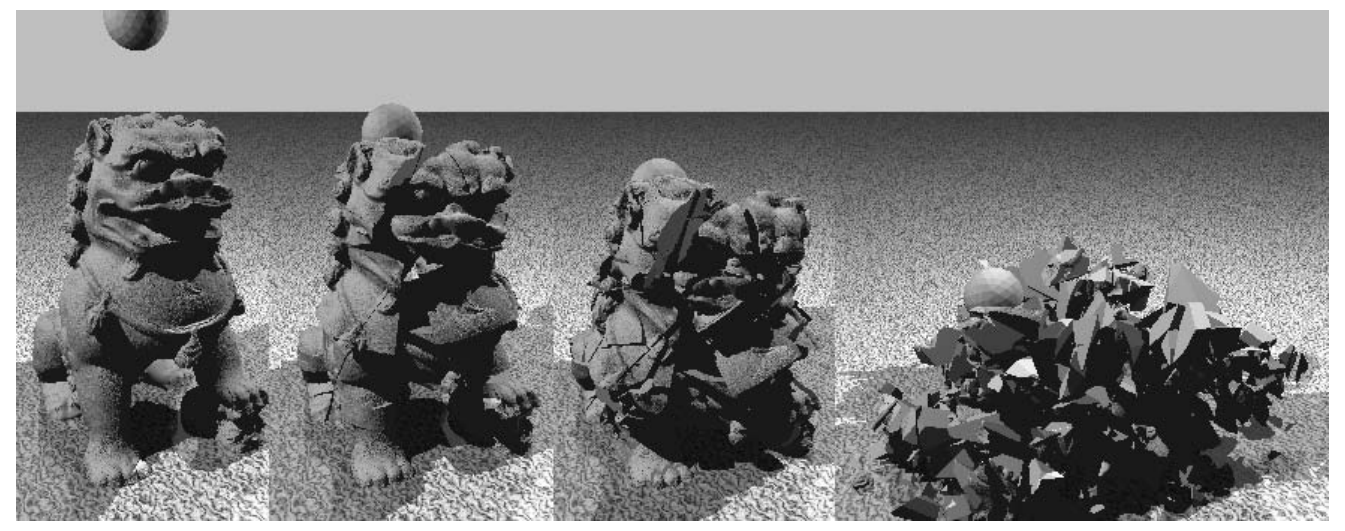

Figure 1: Benchmark Lion: In this simulation, a spherical ball falls on top of a Chinese statue of a lion and the lion gradually breaks into a high number of colliding pieces. This model has $805 \mathrm{~K}$ vertices and $1.6 \mathrm{M}$ triangles. In this scene with changing topologies, our novel collision detection algorithm based on a deforming filter increases the culling efficiency and reduces the number of elementary tests by $10 x$ when compared to prior methods and improves the performance of the CCD algorithm by $4.1 x$.

\begin{abstract}
We present a novel culling algorithm that uses deforming nonpenetration filters to improve the performance of continuous collision detection (CCD) algorithms. The underlying idea is to use a simple and effective filter that reduces both the number of false positives and the elementary tests between the primitives. This filter is derived from the coplanarity condition and can be easily combined with other methods used to accelerate CCD. We have implemented the algorithm and tested its performance on many non-rigid simulations. In practice, we can reduce the number of false positives significantly and improve the overall performance of CCD algorithms by $1.5-8.2 \mathrm{x}$.
\end{abstract}

\section{Introduction}

Continuous collision detection (CCD) is widely used in different applications, including physically-based simulation, CAD/CAM, and robot motion planning. Its main purpose is to check for collisions between two discrete positions of the objects or primitives by using some form of interpolating trajectory. Some algorithms use a linearly interpolating trajectory between the corresponding vertices at two different positions and CCD computations reduce to performing elementary tests between the primitives based on this formulation. Specifically, the CCD test between two deforming triangles reduces to performing 9 vertex-face (VF) and 6 edge-edge

*e-mail: tang_m@zju.edu.cn

†e-mail: dm@cs.unc.edu

‡e-mail: trf@zju.edu.cn
(EE) elementary tests based on coplanarity conditions. Each elementary test can be reduced to computing the roots of a cubic equation [Provot 1997; Bridson et al. 2002].

There is an extensive amount of research on accelerating the performance of CCD algorithms between complex deformable models and reducing the number of elementary tests. Most algorithms use bounding volume hierarchies (BVHs) or a combination of highlevel and low-level culling that use bounds on normals or mesh connectivity or GPU-based accelerations. However, the current CCD algorithms spend a high fraction of the total query time in performing the elementary tests between the primitives [Hutter and Fuhrmann 2007; Curtis et al. 2008; Tang et al. 2009a; Sud et al. 2006; Wong and Baciu 2006] and result in a very high number of false positives (i.e., $95 \%$ or more).

Main Result: In this paper, we present a simple culling algorithm that can significantly reduce the number of false positives in terms of elementary tests and improve the overall performance of CCD algorithms. We introduce a new non-penetration filter which can remove many false positives that cannot be culled by BVHs or other culling methods. The main idea is to exploit the coplanarity condition of the elementary tests and check for overlap between the primitives at every deforming instance of the continuous trajectory between the two discrete time steps. We derive two formulations for this deforming non-penetration filter: one for the VF test and the other for the EE test.

Our approach is complementary to prior CCD algorithms and can be easily combined with BVHs or algorithms that use higher level culling based on normal bounds [Tang et al. 2009a] or lower level culling based on mesh connectivity [Curtis et al. 2008]. We have tested its performance on complex benchmarks corresponding to cloth simulation, breaking objects, and N-body simulations with high model complexity and observed up to $58 \mathrm{x}$ reduction in the number of elementary tests and up to $8.2 \mathrm{x}$ improvement in the performance of the overall CCD algorithm.

Organization: The rest of the paper is organized as follows: Section 2 gives a brief survey of prior work. We introduce our notation 
and describe the coplanarity-based culling method and the deforming non-penetration filter in Section 3. We present the overall CCD algorithm and its implementation in Section 4. We compare our approach with prior algorithms in Section 5.

\section{Related Work}

Many efficient algorithms have been designed for CCD between rigid models and deformable models [Govindaraju et al. 2005; Hutter and Fuhrmann 2007; Zhang et al. 2007; Curtis et al. 2008; Tang et al. 2009a; Tang et al. 2009b]. Some of these approaches linearly interpolate between the vertices of the model and compute the first time-of-contact based on hierarchical culling and performing elementary tests between the triangle pairs. Based on linearly interpolating motion of the vertices, the elementary tests reduce to solving cubic equations. However, these tests can be expensive and also sensitive to tolerance [Brochu and Bridson 2009]. Other CCD formulations use a different formulation of the interpolating motion [Redon et al. 2002; Redon et al. June, 2004; Kim and Rossignac 2003]. Most of these methods reduce to computing roots of polynomial functions and can be accelerated using Béizer clipping [Sederberg and Nishita 1990], recursive subdivision [Taubin 1994], or eigenvalue computations [Manocha and Demmel 1995].

Most of the recent work on CCD algorithms has been on designing high-level and low-level culling algorithms that can reduce the number of elementary tests between the primitives.

High-level culling: Many high-level methods use BVHs to cull away the non-overlapping primitives and they can use tight fitting bounding volumes such as k-DOPs or OBBs to obtain higher culling efficiency. Other culling methods check for self-collisions based on surface normals and curvature [Volino and Thalmann 1994; Provot 1997; Mezger et al. 2003]. Recently, Tang et al. [2009a] extended these ideas to CCD and presented continuous normal cones (CNCs).

Low-level culling: Hutter and Fuhrmann [2007] used bounding volumes (k-DOPs) of the primitives to avoid performing elementary tests between different features. Curtis et al. [2008] and Wong and Baciu [2006] used masking schemes to remove the redundant elementary tests. However, neither adjacent triangles nor their features can be culled by bounding volumes. Govindaraju et al. [2005] eliminate some of the elementary tests associated with adjacent triangles based on some of the tests between the non-adjacent primitives. Tang et al. [2009a] proposed the concept of orphan sets to eliminate almost all the redundant elementary tests between adjacent triangles. The idea has been further extended to procedural representation triangles (PR-Triangles) to remove all redundant elementary tests between non-adjacent triangles. Although these methods can lower the number of elementary tests and false positives, the current CCD algorithms can still result in a high number of false positives (e.g., $95 \%$ or more).

\section{Deforming Non-Penetration Filter}

In this section, we introduce the notation used and present our culling algorithm that reduces the number of false positives.

\subsection{Notations}

We use following notations in the rest of the paper:

- $S V(X)$ is the swept volume of a deforming primitive $X$ along the linear interpolating path between the vertices.

- $B V(X)$ is the bounding volume of a deforming primitive $X$.

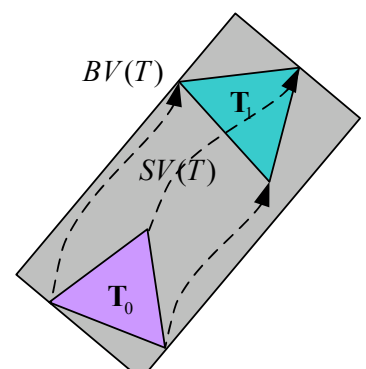

(a) Bounding volume of a deforming triangle

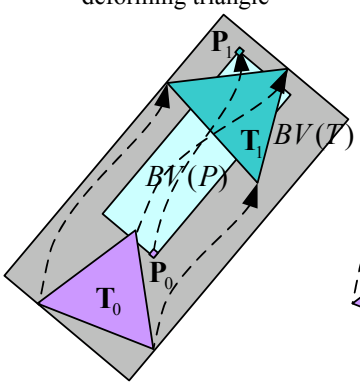

(c) Bounding volume test

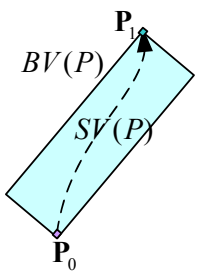

(b) Bounding volume of a deforming vertex

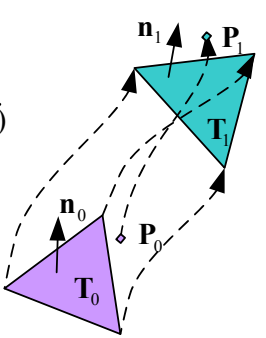

(d) Coplanarity test
Figure 2: Deforming Filter: For a deforming triangle $T$ and $a$ deforming vertex $P$ defined by $T_{0}, T_{1}$ and $P_{0}, P_{1}$ respectively, the bounding volume test $(c)$ becomes quite conservative. The coplanarity test $(d)$ checks whether a penetration between the primitives during the time interval. If the vertex is always on the same side of the triangle during the entire time interval, then the four vertices associated with that elementary test cannot be coplanar during the time interval $[0,1]$ and therefore, no collision occurs.

- $T_{0}, T_{1}$ and $T_{t}$ represent the instances of a deforming triangle $T$ at $t=0, t=1$, and arbitrary $t \in[0,1]$, respectively.

- $a_{0}, b_{0}$, and $c_{0}$ are the three vertices of $T_{0} . a_{1}, b_{1}$, and $c_{1}$ are the vertices of $T_{1} . a_{t}, b_{t}$, and $c_{t}$ are the vertices of $T_{t}$.

- $n_{0}, n_{1}$, and $n_{t}$ are the normal vectors of $T_{0}, T_{1}$, and $T_{t}$.

- $P_{0}, P_{1}$ and $P_{t}$ are the instances of a deforming vertex $P$ at $t=0, t=1$, and arbitrary $t \in[0,1]$, respectively.

- Operator ' $*$ ', ' ', and ' $x$ ' denote multiple of two scale values, dot product of two vectors, and cross product of two vectors, respectively.

\subsection{Motivation}

Given the linear interpolating motion between the vertices, the CCD test between a triangle pair can be reduced to two types of elementary tests: $6 \mathrm{VF}$ tests and $9 \mathrm{EE}$ tests. Each elementary test can be further broken down into two parts: coplanarity test and inside test. Both the VF tests and EE tests involve the use of four deforming vertices, and a necessary condition for a collision is that these four vertices be coplanar. Provot [1997] showed the coplanarity test of four vertices can be reduced to finding roots of a cubic equation. Instead of solving the cubic equation, we will deduce a sufficient condition that these four vertices are non-coplanar during the time interval. By using this condition, many elementary tests can be culled and we do not need to solve the cubic equations.

We use bounding volumes for each primitive (edge, face, vertex), and perform bounding volume based culling before the elementary tests. However, the bounding volume tests can become quite con- 


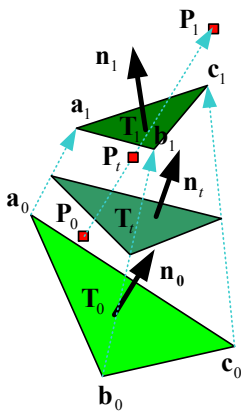

(a) Deforming triangle (T) \& deforming vertex $(\mathbf{P})$

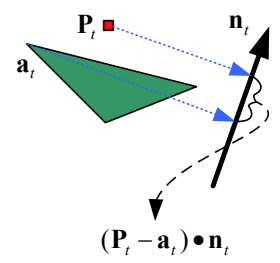

(b) Projected distance between $\mathbf{P}_{t}$ and $\mathbf{T}_{t}$
Figure 3: VF test filter: To perform a VF test between a deforming triangle (defined by $a_{0}, b_{0}, c_{0}$ at $t=0$, and $a_{1}, b_{1}, c_{1}$ at $t=1$ ) and a moving vertex (defined by $P_{0}$ at $t=0$ and $P_{1}$ at $t=1$ ), we need to check for coplanarity between the vertex and the triangle by finding a $t(t \in[0,1])$ when the projected distance along the normal vector of the triangle is equal to zero, i.e., $\left(P_{t}-a_{t}\right) \cdot n_{t}=0$.

servative for primitive level CCD tests. For the example shown in Fig. 2, $B V(T)$ and $B V(P)$ are the bounding volumes of a deforming triangle $T$ and a deforming vertex $P$, respectively. In order to perform culling, $B V(T)$ and $B V(P)$ must contain the swept volumes of deforming primitives, i.e., $S V(T)$ and $S V(P)$, respectively (Fig. 2(a-b)). The VF pair $\{T, P\}$ will be tested for exact $\mathrm{CCD}$ test, since their bounding volumes overlap during the time interval $[0,1]$ (Fig. 2(c)).

We make use of the following observation: if the vertex $P$ is always on the same side of the triangle $T$ along the entire $[0,1]$ trajectory, this pair should be classified as a false positive since it cannot be coplanar (Fig. 2(d)). Based on this observation, we derive a sufficient condition to check whether $P_{t}$ will always be on the same side of $T_{t}$ during the time interval $[0,1]$. If a pair of primitives satisfies this condition, then we don't need to perform the exact test in terms of solving a cubic equation.

The idea can be similarly extended for the EE tests: if there is no crossing between the two deforming edges along the continuous trajectory, they cannot be coplanar during the time interval.

\subsection{Coplanarity Test}

In order to check the coplanarity of a vertex $P_{t}$ and a triangle $T_{t}$, we need to calculate the projected distance between them along the direction of $n_{t}$, as shown in Fig. 3(b). If this distance becomes zero at any time interval, than the four vertices of the two primitives are classified as coplanar.

Non-coplanar Theorem for VF tests: For a triangle $T_{t}$ and $a$ vertex $P_{t}$ defined by the start and end positions during the interval $[0,1]$, these positions are linearly interpolated in the interval with respect to the time variable, $t$. If the following four scalar values: $A, B, \frac{2 * C+F}{3}$, and $\frac{2 * D+E}{3}$ have the same sign, $T_{t}$ and $P_{t}$ will not be coplanar during the interval:

$$
\begin{aligned}
& A=\left(P_{0}-a_{0}\right) \cdot n_{0}, \quad B=\left(P_{1}-a_{1}\right) \cdot n_{1} \\
& C=\left(P_{0}-a_{0}\right) \cdot \hat{n}, \quad D=\left(P_{1}-a_{1}\right) \cdot \hat{n} \\
& E=\left(P_{0}-a_{0}\right) \cdot n_{1}, \quad F=\left(P_{1}-a_{1}\right) \cdot n_{0}
\end{aligned}
$$

And:

$$
\begin{aligned}
n_{0}= & \left(b_{0}-a_{0}\right) \times\left(c_{0}-a_{0}\right), n_{1}=\left(b_{1}-a_{1}\right) \times\left(c_{1}-a_{1}\right) \\
\hat{n}= & \frac{\left(n_{0}+n_{1}-\left(\overrightarrow{v_{b}}-\overrightarrow{v_{a}}\right) \times\left(\overrightarrow{v_{c}}-\overrightarrow{v_{a}}\right)\right)}{2} \\
& \overrightarrow{v_{a}}=a_{1}-a_{0}, \overrightarrow{v_{b}}=b_{1}-b_{0}, \overrightarrow{v_{c}}=c_{1}-c_{0} .
\end{aligned}
$$

Proof. The normal vector $n_{t}$ of the deforming triangle at time $t$ can be represented as following:

$$
n_{t}=n_{0} * B_{0}^{2}(t)+\hat{n} * B_{1}^{2}(t)+n_{1} * B_{2}^{2}(t)
$$

where $B_{i}^{2}(t)$ is the $i^{t h}$ basis function of the Bernstein polynomials of degree 2 .

We define: $\alpha=B_{0}^{2}(t)=(1-t)^{2}, \beta=B_{1}^{2}(t)=2 * t *(1-t)$, and $\gamma=B_{1}^{2}(t)=t^{2}$. Then Equation (4) becomes:

$$
n_{t}=n_{0} * \alpha+\hat{n} * \beta+n_{1} * \gamma
$$

For the moving vertex $P_{t}=P_{0} *(1-t)+P_{1} * t$ and a vertex of the deforming triangle $a_{t}=a_{0} *(1-t)+a_{1} * t$, their projected distant along $n_{t}$ is:

$$
\begin{aligned}
\left(P_{t}-a_{t}\right) \cdot n_{t}= & \left(\left(P_{0}-a_{0}\right) *(1-t)+\left(P_{1}-a_{1}\right) * t\right) \cdot n_{t} \\
= & \left(\left(P_{0}-a_{0}\right) *(1-t)+\left(P_{1}-a_{1}\right) * t\right) \\
& \cdot\left(n_{0} * \alpha+\hat{n} * \beta+n_{1} * \gamma\right) \\
= & \left(P_{0}-a_{0}\right) \cdot n_{0} *(1-t) * \alpha \\
+ & \left(P_{0}-a_{0}\right) \cdot \hat{n} *(1-t) * \beta \\
+ & \left(P_{0}-a_{0}\right) \cdot n_{1} *(1-t) * \gamma \\
+ & \left(P_{1}-a_{1}\right) \cdot n_{1} * t * \gamma \\
+ & \left(P_{1}-a_{1}\right) \cdot \hat{n} * t * \beta \\
+ & \left(P_{1}-a_{1}\right) \cdot n_{0} * t * \alpha
\end{aligned}
$$

Substitute $\alpha, \beta$, and $\gamma$, we have:

$$
\begin{aligned}
\left(P_{t}-a_{t}\right) \cdot n_{t} & =\left(P_{0}-a_{0}\right) \cdot n_{0} *(1-t)^{3} \\
+ & \left(P_{0}-a_{0}\right) \cdot \hat{n} * 2 *(1-t)^{2} * t \\
+ & \left(P_{0}-a_{0}\right) \cdot n_{1} *(1-t) * t^{2} \\
+ & \left(P_{1}-a_{1}\right) \cdot n_{1} * t^{3} \\
+ & \left(P_{1}-a_{1}\right) \cdot \hat{n} * 2 * t^{2} *(1-t) \\
+ & \left(P_{1}-a_{1}\right) \cdot n_{0} * t *(1-t)^{2}
\end{aligned}
$$

Using the symbols defined by Equations(1)-(3), Equation (5) becomes:

$$
\begin{aligned}
\left(P_{t}-a_{t}\right) \cdot n_{t} & =A *(1-t)^{3}+C * 2 *(1-t)^{2} * t \\
& +E *(1-t) * t^{2}+B * t^{3} \\
& +D * 2 * t^{2} *(1-t)+F * t *(1-t)^{2} \\
& =A * B_{0}^{3}(t)+\frac{2 * C+F}{3} * B_{1}^{3}(t) \\
& +\frac{2 * D+E}{3} * B_{2}^{3}(t)+B * B_{3}^{3}(t)
\end{aligned}
$$

where $B_{i}^{3}(t)$ is the $i^{t h}$ basis function of the Bernstein polynomials of degree 3 .

By using the convex hull property associated with control points of the Bernstein basis, the range of the projected distance between $P_{t}$ and $T_{t}$ is bounded by the control vertices. In our case, these control vertices are the four scalar values: $A, B, \frac{2 * C+F}{3}$, and $\frac{2 * D+E}{3}$. 


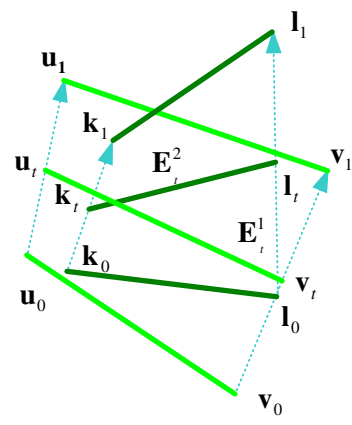

(a) Two deforming edges $\mathbf{E}^{1} \& \mathbf{E}^{2}$

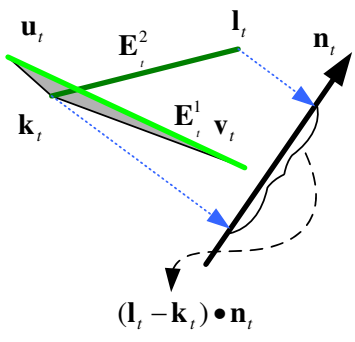
between $\mathbf{E}^{1}$ and $\mathbf{E}^{2}$ (b) Projected distance

Figure 4: EE test filter: To perform a EE test between the two edges $E^{1}$ and $E^{2}$ (defined by $u_{0}, v_{0}$ and $k_{0}, l_{0}$ at $t=0, u_{1}, v_{1}$ and $k_{1}, l_{1}$ at $t=1$ ), we need to check the coplanarity conditions of these vertices by finding all $t(t \in[0,1])$ where the projected distance between $l_{t}$ and the triangle defined by $k_{t}, u_{t}$, and $v_{t}$ is equal to zero, i.e., $\left(l_{t}-k_{t}\right) \cdot n_{t}=0$.

So we obtain the sufficient condition for non-coplanar: If all the four scalar values are negative/positive, the vertices $P_{t}$ will never be on the plane defined by the three vertices $\left(a_{t}, b_{t}\right.$, and $\left.c_{t}\right)$ of the deforming triangle $T_{t}$.

The geometric interpretation of symbol $A$ and $B$ is that they represent the projected distances at $t=0$ and $t=1$, respectively. From Equation( 6), we conclude that it is not sufficient to only check the signs of $A$ and $B$, the values of other symbols $C-F$ also affect the coplanarity condition.

\subsubsection{Extension to EE tests}

In order to perform an elementary test between two edges $E^{1}$ and $E^{2}$ (defined by $u_{0}, v_{0}$ and $k_{0}, l_{0}$ at $t=0, u_{1}, v_{1}$ and $k_{1}, l_{1}$ at $t=$ 1 ), we need to check the coplanarity of these four points (Fig. 4). By replacing $P_{t}, a_{t}, b_{t}, c_{t}$ with $l_{t}, k_{t}, u_{t}, v_{t}$, we can symmetrically deduce a non-coplanar theorem for EE tests:

Non-coplanar Theorem for EE tests: For two deforming edges $E^{1}$ and $E^{2}$ defined by the start and end positions during the interval $[0,1]$, these positions are linearly interpolated in the interval with respect to the time variable, $t$. If the following four scalar values: $A^{\prime}, B^{\prime}, \frac{2 * C^{\prime}+F^{\prime}}{3}$, and $\frac{2 * D^{\prime}+E^{\prime}}{3}$ have the same sign, $E^{1}$ and $E^{2}$ will not be coplanar during the interval:

$$
\begin{aligned}
& A^{\prime}=\left(l_{0}-k_{0}\right) \cdot n_{0}^{\prime}, \quad B^{\prime}=\left(l_{1}-k_{1}\right) \cdot n_{1}^{\prime} \\
& C^{\prime}=\left(l_{0}-k_{0}\right) \cdot \hat{n^{\prime}}, \quad D^{\prime}=\left(l_{1}-k_{1}\right) \cdot \hat{n^{\prime}} \\
& E^{\prime}=\left(l_{0}-k_{0}\right) \cdot n_{1}^{\prime}, \quad F^{\prime}=\left(l_{1}-k_{1}\right) \cdot n_{0}^{\prime} \\
& \text { And: } \quad n_{0}^{\prime}=\left(u_{0}-k_{0}\right) \times\left(v_{0}-k_{0}\right), \quad n_{1}^{\prime}=\left(u_{1}-k_{1}\right) \times\left(v_{1}-k_{1}\right) \\
& \begin{array}{c}
\hat{n^{\prime}}=\frac{\left(n_{0}^{\prime}+n_{1}^{\prime}-\left(\overrightarrow{v_{u}}-\overrightarrow{v_{k}}\right) \times\left(\overrightarrow{v_{v}}-\overrightarrow{v_{k}}\right)\right)}{\overrightarrow{v_{k}}=} k_{1}-k_{0}, \overrightarrow{v_{u}}=u_{1}-u_{0}, \overrightarrow{v_{v}}=v_{1}-v_{0} .
\end{array}
\end{aligned}
$$

\subsection{CCD Algorithm}

We use the non-planarity filter defined above to perform coplanarity-based culling. We use Equations(1)-(3) to calculate the values of the four control vertices defined in those equations for a primitive pair. If these variables have the same sign, this pair will be identified as a false positive.

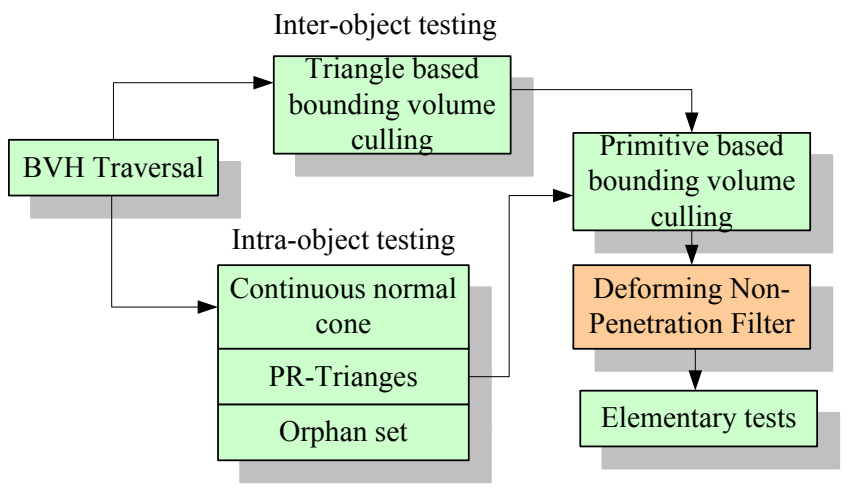

Figure 5: CCD algorithm with deforming non-penetration filter: The deforming non-penetration filter is complementary to the overall CCD algorithm and can be performed right before the elementary tests.

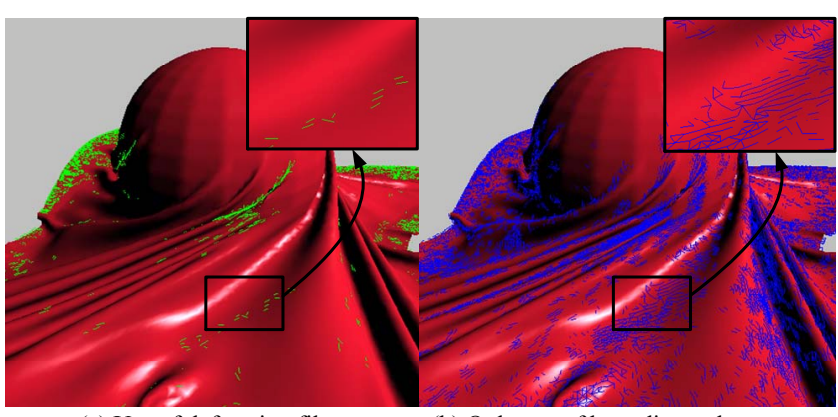

(a) Use of deforming filter

(b) Only use of bounding volume tests

Figure 6: Edges involved by EE tests with different culling methods: This figure shows edges (highlighted in green and blue respectively) used by EE tests during a specific frame of the Cloth benchmark $(F i g .7(d))$ with coplanarity-based culling and bounding volume based culling respectively. Comparing to only bounding volume based culling, the number of EE elementary tests are reduced by $80 \%$ with the deforming non-penetration filter.

The deforming non-penetration filter can be combined with hierarchical representations (Fig. 5). During the traversal of BVH, we perform different high-level culling methods to remove redundant pairwise tests or primitive pairs that are not in close proximity to one another. Next, the deforming non-penetration filter is used as part of low-level culling to further remove the false positives.

Fig. 6 compares the effects of bounding volume based culling and coplanarity-based culling. The green line segments in Fig. 6(a) and blue line segments in Fig. 6(b) describe the distribution of edges involved in EE tests with deforming non-penetration filter and bounding volume based culling respectively. As shown in the figure, compared to the number of tests in the bounding volume based culling, we observe more than $80 \%$ reduction in the number of elementary tests on this scene.

\section{Implementation and Performance}

In this section, we describe our implementation and highlight the performance of our algorithm on several benchmarks. 


\subsection{Implementation}

We have implemented our algorithm on a standard $2.4 \mathrm{GHz}$ Intel Pentium machine with 4GB RAM on 32-bit Windows/XP platform. The performance is measured using a single thread. We use k-DOPs (specifically 16-DOPs) as bounding volumes because they provide a good balance between tight fitting and rapid updating.

We use restructuring and refitting to update the hierarchy for deformable models. Intel SSE/SSE2 instructions are used to accelerate the updating of and overlap testing between bounding volumes. We use an implementation of the ICCD algorithm based on normal cones for high-level culling [Tang et al. 2009a] as a baseline for comparison. We also integrate our filters with the R-Triangle algorithm [Curtis et al. 2008], as it spends a large fraction of the query time on the elementary tests.

\subsection{Benchmarks \& Performance}

In order to test the performance of our algorithm, we used six different benchmarks, arising from different simulations with different characteristics.

- Lion: For this breaking benchmark with 1.6M triangles (Figure 1), our algorithm reduces the number of elementary test by $10 \mathrm{x}$ as compared to prior CCD algorithms.

- Balls: A scene with hundreds of balls (34K triangles) that are colliding with each other (Figure 7(a)). Our deforming nonpenetration filter reduces the number of elementary tests by $17 \mathrm{x}$.

- Falling Alphabets: Multiple deforming characters ( $5 K$ triangles) fall into a bowl and break into pieces (Figure 7(b)). Our CCD algorithm reduces the number of elementary tests by $58 x$.

- Princess: This model ( $40 K$ triangles) has many inter- and intra-object collisions (Figure 7(c)). Our algorithm reduces the number of elementary tests by $20.5 x$.

- Cloth: A cloth (92K triangles) has a high number of selfcollisions (Figure 7(d)). Our algorithm reduces the number of elementary tests by $11.5 \mathrm{x}$.

- Flamenco: This benchmark ( $49 K$ triangles) has many interand intra-object collisions (Figure 7(e)). The CCD algorithm with the deforming non-penetration filter reduces the number of elementary tests by $17 x$.

Fig. 8 highlights the culling efficiency of our algorithm by comparing the number of elementary tests performed. As shown in the figure, the elementary tests are dramatically reduced by $10-58.7 \mathrm{x}$. Also, by preventing the computation of these false positives, we achieve $1.5-3.5 \mathrm{x}$ improvement on overall performance in comparison with the ICCD algorithm [Tang et al. 2009a], and $2.4-8.2 \mathrm{x}$ improvement over R-Triangles [Curtis et al. 2008](Table. 1). For Benchmark Flamenco, its average running time per frame in RTriangles, ICCD, and our system are shown in Figure 9.

\section{Analysis and Comparison}

In this section, we analyze our results and compare against prior methods.

\subsection{Analysis}

Although the performance of our deforming non-penetration filter varies with different benchmarks, we are able to obtain high culling

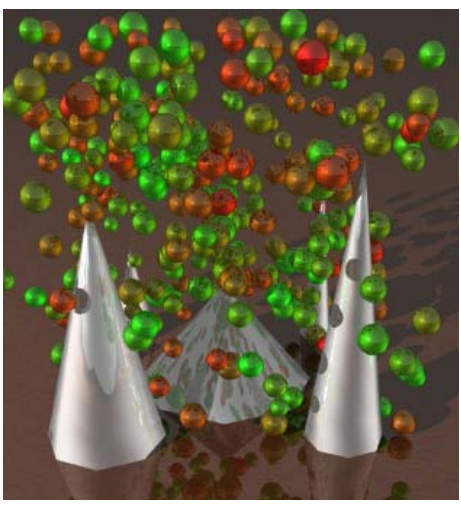

(a) Balls

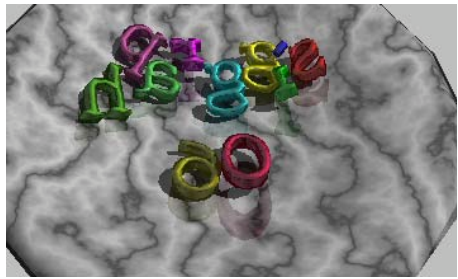

(b) Falling Alphabets

(c) Princess

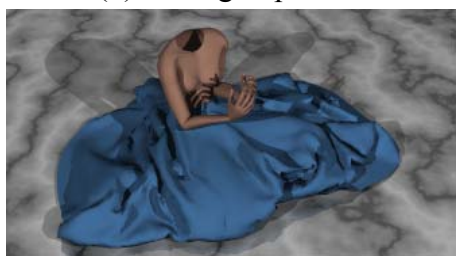

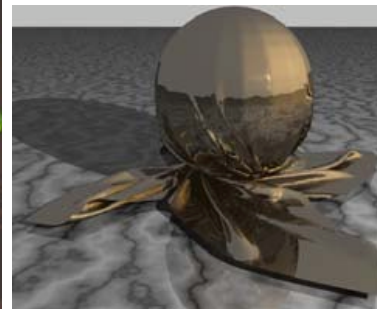

(d) Cloth

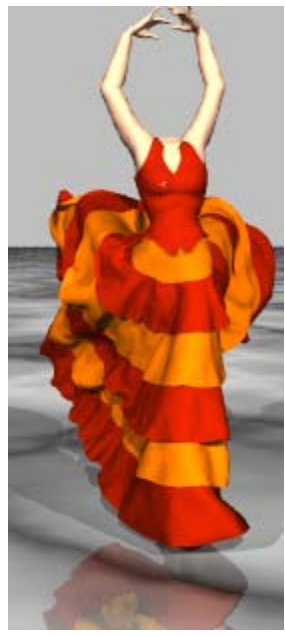

(e) Flamenco
Figure 7: Benchmarks: All the benchmarks have multiple simulation steps. We perform CCD, including self-collisions, between discrete steps of the simulation and compute the first time-of-contact.

efficiency in many of them. The basic filter test proposed in the noncoplanar theorems is conservative. Intuitively, our non-coplanar theorem eliminates the need to perform exact tests on pairs where no 'penetrations' occur along the continuous trajectory. In practice, due to the temporal coherence between subsequent frames, the 'penetrations' only occur infrequently. Fig. 10 shows the changing of ratios of primitive pairs in which no 'penetration' occurs during the time interval. In the figure, the ratios stay above $80 \%$, and are changing smoothly. Due to coherence, our deforming nonpenetration filter can be quite effective.

We use the Interval-Newton method to solve the cubic equations. An elementary test takes about roughly 155 additions, 217 multiplications, and 6 divisions on average (all for float point values). On the other hand, the deforming non-penetration filter only need to perform 29 additions and 40 multiplications on average. In practice, the speed of a deforming non-penetration filter is about 5.5-10.2x faster than an exact test.

\subsection{Comparison}

In this section, we compare our novel algorithm with prior culling algorithms.

Bounding volume based culling: Bounding volumes (e.g. spheres [Hubbard 1993; Palmer and Grimsdale 1995; Bradshaw and O'Sullivan 2004], AABBs [van den Bergen 1997], k- 


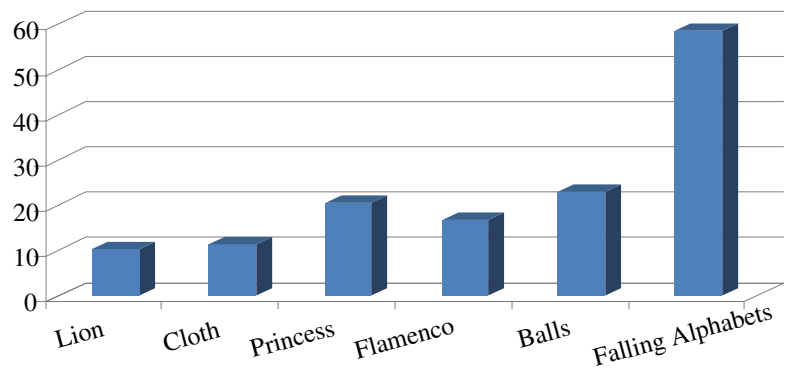

Figure 8: Culling efficiency: This figure shows the culling efficiency of our algorithm by comparing the number of elementary tests performed. For the benchmarks, our algorithm is capable of reducing the number of elementary tests by $10-58.7 x$.

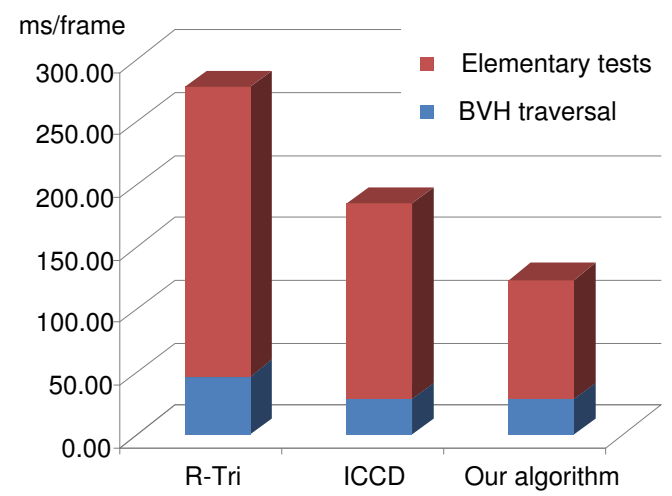

Figure 9: Average running time per frame: This figure shows the average running time per frame of Benchmark Flamenco with $R$-Triangles [Curtis et al. 2008], ICCD [Tang et al. 2009a], and our algorithm, respectively.

DOPs [Klosowski et al. 1998], OBBs [Gottschalk et al. 1996], etc.) can be used to remove primitive pairs whose primitives are not in close proximity to each other. These methods work for inter-object and intra-object collisions. But for CCD, the bounding volumes of primitives are quite conservative and make false positive rates very high. Our deforming non-penetration filter can improve the culling efficiency.

Orphan sets: Orphan sets [Tang et al. 2009a] are quite effective at removing redundant elementary tests between adjacent triangles. However, they do not reduce the false positives between non-adjacent primitives, whereas our filter can be used for these cases.

Continuous normal cones: CNCs [Tang et al. 2009a] are applied to remove false positives on large areas where are relatively flat in a mesh. They only work for self-collisions, and are quite conservative on models with high curvatures.

Representative triangles: Representative triangles [Curtis et al. 2008] can remove all the redundant elementary tests that are caused by shared features between the adjacent triangles. Procedural representative triangles [Tang et al. 2009a] combine it with CNC culling. Our deforming non-penetration filter complements these methods.

GPU based culling: GPU algorithms that use occlusion queries [Govindaraju et al. 2005] and rasterization based distance field computation [Sud et al. 2006] are complementary to our culling algorithm.
Table 1: Performance and Speedup: This table shows the average query time of our method and performance improvement over ICCD [Tang et al. 2009a] and R-Triangles [Curtis et al. 2008].

\begin{tabular}{|l|c|c|c|}
\hline Model & $\begin{array}{c}\text { Query } \\
\text { (time } m s \text { ) }\end{array}$ & $\begin{array}{c}\text { Speedup over } \\
\text { ICCD }\end{array}$ & $\begin{array}{c}\text { Speedup over } \\
\text { R-Triangles }\end{array}$ \\
\hline \hline Cloth & 144 & $2 \mathrm{x}$ & $2.4 \mathrm{x}$ \\
\hline Princess & 18.7 & $2.4 \mathrm{x}$ & $3.1 \mathrm{x}$ \\
\hline Flamenco & 110 & $1.5 \mathrm{x}$ & $2.8 \mathrm{x}$ \\
\hline Balls & 52.8 & $1.7 \mathrm{x}$ & $3.4 \mathrm{x}$ \\
\hline $\begin{array}{l}\text { Falling } \\
\text { Alphabets }\end{array}$ & 4.6 & $3.5 \mathrm{x}$ & $8.2 \mathrm{x}$ \\
\hline Lion & 4432 & $2.4 \mathrm{x}$ & $4.1 \mathrm{x}$ \\
\hline
\end{tabular}

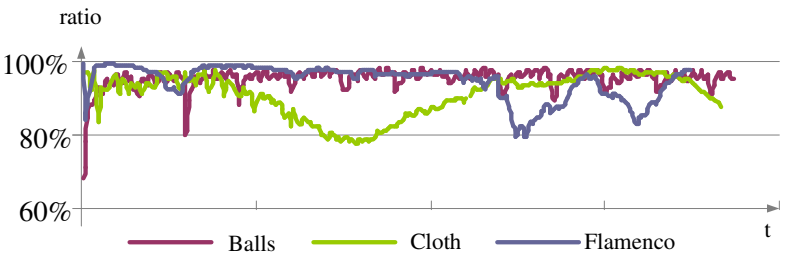

Figure 10: Temporal coherency: $(a),(b)$, and $(c)$ show the changing ratios of primitive pairs in which no penetration occurs during the time interval for the benchmarks Balls, Cloth, and Flamenco, respectively. The ratios tend to be above $80 \%$, and are changing smoothly.

\subsection{Limitations}

Our approach only provides a filter at the feature level. As a result, it needs to be applied for each VF or EE test. Moreover, our test can be conservative due to the underlying formulation. If a high-level culling algorithm is able to cull away a high percentage of false positives, then we may obtain a relatively small improvement using our deforming non-penetration filter.

\section{Conclusion and Future Work}

We have presented a novel culling algorithm for CCD between complex deformable models by proposing a deformable nonpenetration filter. By checking the coplanarity of the vertices of the primitives, our algorithm can significantly reduce the number of false positives, and subsequently improve the overall performance of CCD algorithms. Moreover, our approach is complementary to and can be combined with most prior methods. We test the performance on different benchmarks and observed considerable improvement in terms of reducing the number of false positives.

In our future work, it is interesting to develop enhanced filters that would further improve the culling efficiency. It may also be possible to further improve the performance of the filters by further utilizing temporal coherence and/or using SSE instructions. Currently, our CCD formulation is based on linear movements of vertices, it is possible to derive similar bounds for other motions of the vertices, e.g. arbitrary interplaiting motion [Redon et al. June, 2004]. Finally, we would like to use our CCD algorithm with some well known game Physics engines (e.g., Bullet, Hovak, PhysX, etc.), and also apply our CCD algorithm to more complex benchmarks. 


\section{Acknowledgments}

We would like to thank Jieyi Zhao, Jiang Lin, Peng Du and Ming Lin for useful discussions and the benchmarks. The cloth models were provided by Rasmus Tamstorf at Disney Animation.

This research is supported in part by National Basic Research Program of China (No. 2006CB303106), MOE-Intel special research fund on information technology (No. MOE-INTEL-09-05), ARO Contract W911NF-04-1-0088, NSF awards 0636208, 0917040 and 0904990, DARPA/RDECOM Contract WR91CRB-08-C-0137, and Intel. Tang is supported in part by Natural Science Foundation of China (No. 60803054), National Key Technology R\&D Program, China (No. 2006BAF01A45-05), Natural Science Foundation of Zhejiang, China (No. Y107403), Doctoral subject special scientific research fund of Education Ministry of China (No. 20070335074).

\section{References}

Bradshaw, G., AND O'Sullivan, C. 2004. Adaptive medialaxis approximation for sphere-tree construction. ACM Trans. on Graphics 23, 1, 1-26.

Bridson, R., FEDKiw, R., AND Anderson, J. 2002. Robust treament for collisions, contact and friction for cloth animation. Proc. of ACM SIGGRAPH, 594-603.

BROCHU, T., AND BRIDSON, R. 2009. Numerically robust continuous collision detection fro dynamic explict surfaces. Tech. rep., TR-2009-03, University of British Columbia, Vancouver.

Curtis, S., Tamstorf, R., and Manocha, D. 2008. Fast collision detection for deformable models using representativetriangles. In SI3D '08: Proceedings of the 2008 Symposium on Interactive $3 D$ graphics and games, 61-69.

GotTschalk, S., Lin, M. C., And Manocha, D. 1996. Obbtree: a hierarchical structure for rapid interference detection. In SIGGRAPH '96: Proceedings of the 23rd annual conference on Computer graphics and interactive techniques, ACM, New York, NY, USA, 171-180.

Govindaraju, N., Knott, D., Jain, N., Kabul, I., TAMStorf, R., Gayle, R., Lin, M., And Manocha, D. 2005. Interactive collision detection between deformable models using chromatic decomposition. ACM Trans. on Graphics (Proc. of ACM SIGGRAPH) 24, 3, 991-999.

Hubbard, P. M. 1993. Interactive collision detection. In Proceedings of IEEE Symposium on Research Frontiers in Virtual Reality.

Hutter, M., And Fuhrmann, A. 2007. Optimized continuous collision detection for deformable triangle meshes. In Proc. WSCG '07, 25-32.

KIM, B., AND RossignaC, J. 2003. Collision prediction for polyhedra under screw motions. In ACM Symposium in Solid Modeling and Applications, ACM Press, 4-10.

Klosowski, J., Held, M., Mitchell, J., SowizRal, H., AND ZIKAN, K. 1998. Efficient collision detection using bounding volume hierarchies of k-dops. IEEE Trans. on Visualization and Computer Graphics 4, 1, 21-37.

Manocha, D., And Demmel, J. 1995. Algorithms for intersecting parametric and algebraic curves ii: multiple intersections. Graph. Models Image Process. 57, 2, 81-100.
Mezger, J., Kimmerle, S., And Etzmu $\beta$, O. 2003. Hierarchical techniques in cloth detection for cloth animation. Journal of WSCG 11, 1, 322-329.

PAlmer, I. J., AND GRIMSDale, R. L. 1995. Collision detection for animation using sphere-trees. Computer Graphics Forum 14, $2,105-116$.

Provot, X. 1997. Collision and self-collision handling in cloth model dedicated to design garment. Graphics Interface, 177189.

Redon, S., Kheddar, A., And Coquillart, S. 2002. Fast continuous collision detection between rigid bodies. Proc. of Eurographics (Computer Graphics Forum) 21, 3, 279-288.

Redon, S., Kim, Y. J., Lin, M. C., And Manocha, D. June, 2004. Fast continuous collision detection for articulated models. In SM '04: Proceedings of the ninth ACM symposium on Solid modeling and applications, 145-156.

SAnNA, A., AND Milani, M. 2004. CDFast: an algorithm combining different bounding volume strategies for real time collision detection. SCI Proceedings 2, 144-149.

SederberG, T. W., AND Nishita, T. 1990. Curve intersection using be'zier clipping. Comput. Aided Des. 22, 9, 538-549.

Sud, A., Govindaraju, N., Gayle, R., Kabul, I., And MANOCHA, D. 2006. Fast proximity computation among deformable models using discrete voronoi diagrams. Proc. of ACM SIGGRAPH, 1144-1153.

Tang, M., Curtis, S., Yoon, S.-E., And Manocha, D. 2009. ICCD: Interactive continuous collision detection between deformable models using connectivity-based culling. IEEE Transactions on Visualization and Computer Graphics 15, 4, 544-557.

TAng, M., Kim, Y. J., And Manocha, D. 2009. C2a: Controlled conservative advancement for continuous collision detection of polygonal models. Proceedings of International Conference on Robotics and Automation, 849-854.

TAUBIN, G. 1994. Rasterizing algebraic curves and surfaces. IEEE Comput. Graph. Appl. 14, 2, 14-23.

VAN DEN BERGEN, G. 1997. Efficient collision detection of complex deformable models using AABB trees. Journal of Graphics Tools 2, 4, 1-14.

Volino, P., And Thalmann, N. M. 1994. Efficient selfcollision detection on smoothly discretized surface animations using geometrical shape regularity. Computer Graphics Forum (EuroGraphics Proc.) 13, 3, 155-166.

WonG, W. S.-K., AND BACIU, G. 2006. A randomized marking scheme for continuous collision detection in simulation of deformable surfaces. Proc. of ACM VRCIA, 181-188.

Zhang, X., Redon, S., Lee, M., and Kim, Y. J. 2007. Continuous collision detection for articulated models using taylor models and temporal culling. ACM Transactions on Graphics (Proceedings of SIGGRAPH 2007) 26, 3, 15. 